\title{
Terapia ocupacional em disfunção física: discutindo a produção bibliográfica brasileira no período de 1999 a 2005
}

\section{Occupational therapy in physical dysfunction: discussing the brazilian bibliographical production from 1999 to 2005}

\author{
Taisa Gomes Ferreira ${ }^{1}$, Fátima Corrêa Oliver $^{2}$
}

FERREIRA, T. G.; OLIVER, F. C. Terapia ocupacional em disfunção física: discutindo a produção bibliográfica brasileira no período de 1999 a 2005. Rev. Ter. Ocup. Univ. São Paulo, v. 17, n. 3, p. 108114, set./dez., 2006.

\begin{abstract}
RESUMO: Trata-se de estudo da bibliografia brasileira de terapia ocupacional em disfunção física realizado por levantamento de teses, dissertações, artigos e resumos de trabalhos em congressos (período de 1999 a 2005). Foram consultados: Literatura Latino-Americana e do Caribe de Ciências da Saúde LILACS, Scientific Eletronic Library Online - SciELO e banco de teses da Coordenação de Aprimoramento de Pessoal de Nível Superior - CAPES. Para os resumos foram acessados Anais dos VII e IX Congressos Brasileiros e do VIII Congresso Brasileiro e V Congresso Latinoamericano de Terapia Ocupacional. Foram identificados livros da área, bem como manuais editados (2001 a 2005). Para os artigos também foram consultados os Cadernos de Terapia Ocupacional da Universidade Federal de São Carlos. Foram localizados 213 textos que tratavam do tema. Nesse universo os resumos apresentados em congressos significaram $64 \%$ da produção, enquanto que os artigos, teses e livros representaram $20 \%$, $9 \%$ e $7 \%$ respectivamente. Em relação aos resumos publicados notou-se ligeira tendência de crescimento no período, o que não ocorreu com os artigos, que aumentaram no período de 1999 a 2002 e posteriormente apresentaram tendência de diminuição, com crescimento para o número de livros publicados. No que diz respeito a teses e dissertações o maior número foi publicado em 1999, 2003 e 2004. Observou-se a constituição de um núcleo de discussão com temáticas prevalentes sobre intervenções para ampliar capacidade funcional/ motora, independência/autonomia, bem como a preocupação com análise de contexto, saúde mental da pessoa com deficiência física e metodologia de intervenção entre outras.
\end{abstract}

DESCRITORES: Terapia ocupacional. Bibliografia (Tipo de publicação). Reabilitação. Pessoas portadoras de deficiência/reabilitação.

\footnotetext{
${ }^{1}$ Terapeuta ocupacional. Mestranda do Programa de Ciências da Reabilitação do Depto. de Fisioterapia, Fonoaudiologia e Terapia Ocupacional da FMUSP

2 Terapeuta ocupacional. Docente do Depto Fisioterapia, Fonoaudiologia e Terapia Ocupacional da FMUSP. Doutora em Saúde Pública.

Endereço para correspondência: Departamento de Fisioterapia, Fonoaudiologia e Terapia Ocupacional da FMUSP. Rua Cipotânea, 51. CEP: 05360-000. São Paulo, SP.
} 


\section{INTRODUÇÃO}

$\mathrm{E}$ ste estudo busca compreender como os terapeutas ocupacionais brasileiros têm fundamentado sua prática profissional no acompanhamento de pessoas com disfunções físicas, isto porque a terapia ocupacional junto a esse segmento da população é parte constituinte da história da profissão, estruturada no diálogo com o campo multiprofissional e interdisciplinar em reabilitação.

Desde o surgimento da profissão, o terapeuta ocupacional fez parte da equipe de reabilitação e tem participado da construção de alternativas assistenciais que se estruturaram a partir de diferentes noções de indivíduo, corpo, intervenção técnica, processo de saúde-doençadeficiência-incapacidade, de tratamento e reabilitação. Um dos eixos estruturadores das práticas de reabilitação e de terapia ocupacional em disfunção física tem sido a noção de corpo, que, na maior parte das vezes, é considerado como "máquina", que necessita de adaptação, de reconstrução da função para que sua fragilidade diminua e para que, progressivamente, possa ser alcançada a independência da pessoa para o desenvolvimento das atividades cotidianas de autocuidado, trabalho ou lazer. Essa independência conquistada levaria a maiores possibilidades de inserção e participação na vida social, um dos principais problemas enfrentados também pelas pessoas com disfunções físicas.

Nas últimas décadas tem se observado uma tendência, na literatura da área e nas discussões profissionais, de ampliação do papel profissional e dos desafios colocados para a prática assistencial.

Assim, a própria Federação Mundial de Terapeutas Ocupacionais considera que a profissão deve ter por objetivo contribuir para promover a saúde e o bem estar através da ocupação, facilitando processos de participação das pessoas com incapacidades nas atividades da vida diária e/ou promovendo mudanças ambientais favorecedoras da participação. Isto poderia ser viabilizado pela formação profissional ancorada em diferentes campos do conhecimento, que possibilita aos profissionais trabalhar colaborativamente com pessoas ou grupos, que apresentem problemas na estrutura corporal ou no desenvolvimento das funções, sejam eles consequiências de doenças ou de barreiras à participação (geográficas arquitetônicas ou psicossociais). Seria objetivo dos profissionais identificar a natureza dessas barreiras (ambientais, sociais ou legais) e estruturar alternativas para promover mudanças ambientais e pessoais promotoras de participação. As práticas assistenciais poderiam ser desenvolvidas em diferentes instituições (hospitais, centros de saúde, domicílios, locais de trabalho, escolas entre outras). Ao mesmo tempo, seria objetivo da terapia ocupacional buscar a participação ativa das pessoas acompanhadas no processo terapêutico para possibilitar resultados reais (WFOT, 2005).

Dessa maneira, se observa uma tendência a considerar que o olhar do terapeuta ocupacional não deva projetar-se apenas para o corpo físico das pessoas que acompanha, mas também para o ambiente no qual esta está integrada, problematizando sua qualidade de vida e possibilidades de participação na vida social.

Essa perspectiva pode ser contraditória e conflituosa com posturas profissionais e institucionais, que fomentem tanto a formação como a atuação especializada, seja em técnicas e recursos terapêuticos, seja em instituições que priorizam o acompanhamento de pessoas com determinadas patologias, como tem sido usual no desenvolvimento das instituições de reabilitação e de atenção em saúde no país. Essas tendências têm levado a que os profissionais recém ingressados no mercado de trabalho procurem complementar sua formação buscando cursos de especialização e/ou de atualização em técnicas e recursos terapêuticos específicos, que, inicialmente apóiam e fundamentam a atividade profissional e, posteriormente, podem levar a um certo esgotamento dessa perspectiva de atuação, pois, por vezes, os objetivos primordiais do processo terapêutico ocupacional (aumento da independência, da autonomia ou a ampliação da participação social) não estão diretamente vinculados aos recursos especializados de tratamento, mas à superação de obstáculos (ambientais, sociais e culturais) colocados à participação das pessoas com disfunções físicas.

A experiência profissional de uma das autoras, que durante seis anos atuou em diferentes instituições de reabilitação física (centro de reabilitação, clínicas especializadas, hospital e ambulatórios), bem como na atenção domiciliar, possibilitou a reflexão sobre a importância e papel das técnicas e recursos terapêuticos especializados no desenvolvimento da prática de terapia ocupacional em disfunção física. Essa experiência profissional também favoreceu a reflexão sobre a condição de participação das pessoas com disfunções físicas no processo terapêutico, na definição de objetivos de tratamento/acompanhamento. Por outro lado, tem possibilitado pensar sobre de que maneira se estruturam os processos de avaliação e de definição de objetivos a serem atingidos, pois muitos procedimentos normatizados e estandartizados inviabilizam a escuta e percepção das pessoas com disfunções físicas e suas necessidades de acompanhamento. A maior parte das instituições e serviços de reabilitação, a que uma das autoras esteve vinculada, 
orienta sua prática assistencial considerando a disfunção física como um fator ligado, estritamente ao corpo físico, que deve ser normatizado e regulado dificultando a que profissionais implementem outras perspectivas de atenção.

Tendo em vista essas questões e considerando a recente ampliação do número de profissionais graduados e de escolas de terapia ocupacional no país as autoras consideraram oportuno conhecer as reflexões realizadas por profissionais da área no desenvolvimento da terapia ocupacional com pessoas com disfunção física.

\section{PROCEDIMENTOSMETODOLÓGICOS}

A pesquisa foi baseada no estudo da produção bibliográfica nacional em terapia ocupacional em disfunção física, no período de 1999 a 2005. Considerou-se produção bibliográfica da área as dissertações e teses defendidas, os artigos publicados, os livros editados e os resumos de trabalhos apresentados em congressos nacionais de terapia ocupacional.

Para levantamento dessa produção foram consultadas: a Literatura Latino-Americana e do Caribe de Ciências da Saúde - LILACS, a Scientific Eletronic Library Online SciELO ambos entre 1999 e 2005 e o banco de teses da Coordenação de Aprimoramento de Pessoal de Nível Superior-CAPES, com dados disponíveis de 1999 a 2004. Nessas bases de dados foram utilizados descritores de assunto "Terapia Ocupacional" e "Reabilitação" e palavras do título "Terapia Ocupacional", "Disfunção Física" e "Reabilitação". Para os resumos foram acessados os anais dos VII e IX Congressos Brasileiros de Terapia Ocupacional em Porto Alegre e Recife, respectivamente, bem como do VIII Congresso Brasileiro e V Congresso Latinoamericano de Terapia Ocupacional realizado em Foz do Iguaçú. Foram identificados livros da área, capítulos de livros, bem como manuais editados no período de 1999 a 2005, em bibliotecas e acervos pessoais. Para os artigos também foram consultados os Cadernos de Terapia Ocupacional da Universidade Federal de São Carlos e a Revista de Terapia Ocupacional da Universidade de São Paulo, entre 1999 e 2004, pois no momento de levantamento de dados não estavam disponíveis os Cadernos de Terapia Ocupacional da UFSCar do ano de 2005.

O levantamento de resumos de trabalho de congressos, artigos, dissertações, teses e livros foi seguido de sua localização e obtenção.

A partir da localização dos textos foram realizadas as leituras exploratórias e seletivas do material, a fim de eleger mais detalhadamente os documentos pertinentes ao estudo (GIL, 1996, p. 66-7; PÁDUA, 2002, p. 52-8).
Como orientam Gil (1996) e Pádua (2002) a organização do material bibliográfico subsidiou as leituras analíticas e interpretativas dos textos. Na leitura analítica buscou-se conhecê-los na íntegra, identificar os temas principais e secundários subsidiando sua leitura interpretativa.

\section{RESULTADOSE DISCUSSÃO}

Os textos foram analisados em sua distribuição temporal e por tipo de produção bibliográfica, bem como foram analisados após leitura de seus resumos, buscando conhecer o tema de trabalho apresentado pelos autores, ou seja, o que consideraram relevante para o desenvolvimento e as propostas de intervenção sugeridas tanto para compreender as necessidades das pessoas acompanhadas como para conduzir o tratamento desenvolvido.

No Gráfico 1 estão apresentados os primeiros resultados do levantamento, entre os 213 trabalhos que tratavam do tema, os resumos apresentados em congressos representaram a maior parte da produção, seguidos pelos artigos, teses e livros respectivamente.

Gráfico 1 - Produção bibliográfica, segundo tipo de vublicacão entre 1999 e 2005.
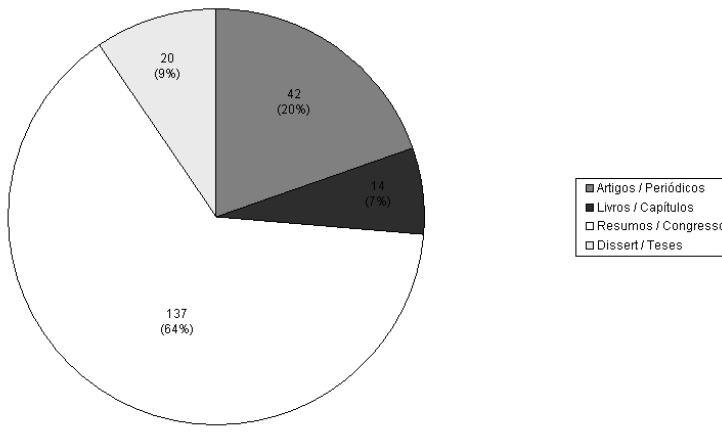

A distribuição temporal da produção bibliográfica apresentada no Gráfico 2 demonstra uma tendência de ascensão na produção de artigos sobre o tema entre 1999 e 2002, observada também na produção de artigos por terapeutas ocupacionais em geral, pois esse período coincide com a finalização dos mestrados de profissionais (BARROS; OLIVER, 2003). No entanto, em 2003 e 2005 se observou decréscimo no número de artigos sobre o tema e ligeiro aumento em 2004. O período de decréscimo nos artigos coincidiu com o de aumento do número de livros e manuais editados no ano de 2004. A não inclusão de artigos dos periódicos da área em 2005 reduziu os artigos publicados em 2005 a aqueles publicados por profissionais em outros periódicos indexados na base LILACS e SciELO, nessas bases os periódicos que têm publicado 
artigos sobre a temática de estudo foram Temas de Desenvolvimento, Revista Brasileira de Reumatologia,
Revista de Fisioterapia, Acta Fisiátrica entre outras, além das revistas específicas de terapia ocupacional.

Gráfico 2 - Produção bibliográfica entre 1999 e 2005, segundo ano e tipo de publicação.

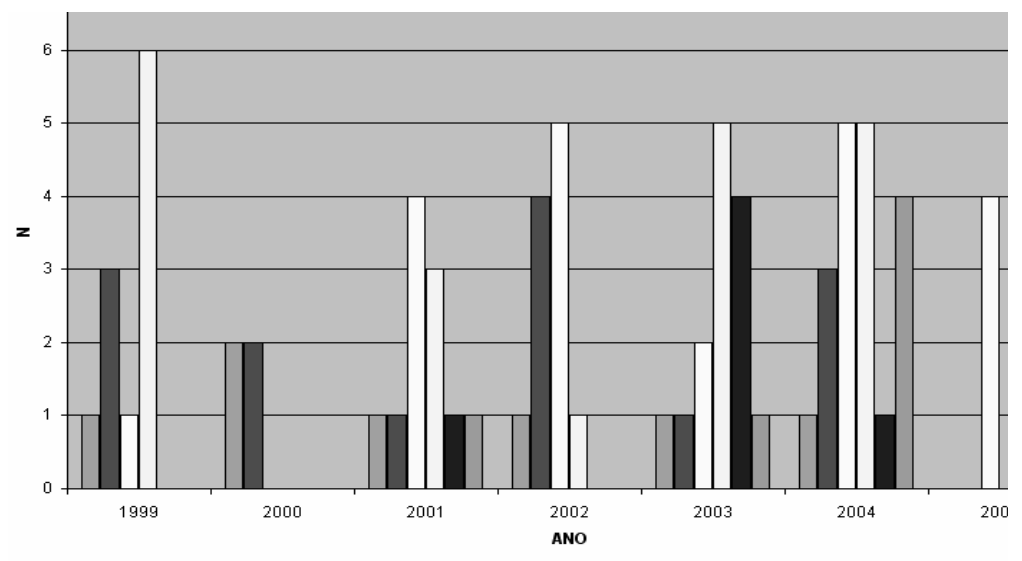

As dissertações e teses estavam concentradas em 1999, 2003 e 2004, apesar dos números absolutos serem pequenos $(\mathrm{n}=20)$, mas serem significativos para a produção acadêmica de uma área em constituição.

No Gráfico 3 em que se apresenta a produção bibliográfica da área, segundo a temática da disfunção física e tipos de publicação observa-se que os resumos de trabalhos publicados em anais, que significaram $64 \%$ da produção bibliográfica, representaram apenas $10,1 \%$ dos apresentados no Congresso de 2001, 18\% dos apresentados em 2003 e 13,8\% dos apresentados no Congresso de 2005. Isto indicaria pequena presença de trabalhos de profissionais que acompanham pessoas com disfunção física nos congressos nacionais, campo de intervenção importante para a constituição histórica da terapia ocupacional em reabilitação. Ou talvez maior dificuldade de profissionais vinculados a essa temática para se deslocar para a capital em que se realizou o evento nacional em 2005, já que se observou um aumento do número de trabalhos sobre o tema apresentado entre os congressos de 2001 e 2003. Por outro lado, os profissionais podem estar apresentando trabalhos também em outros congressos de interesse para o campo e que não foram acessados neste estudo.

Gráfico 3 - Produção bibliográfica, segundo tipo de publicação e temática, entre 1999 e 2005.

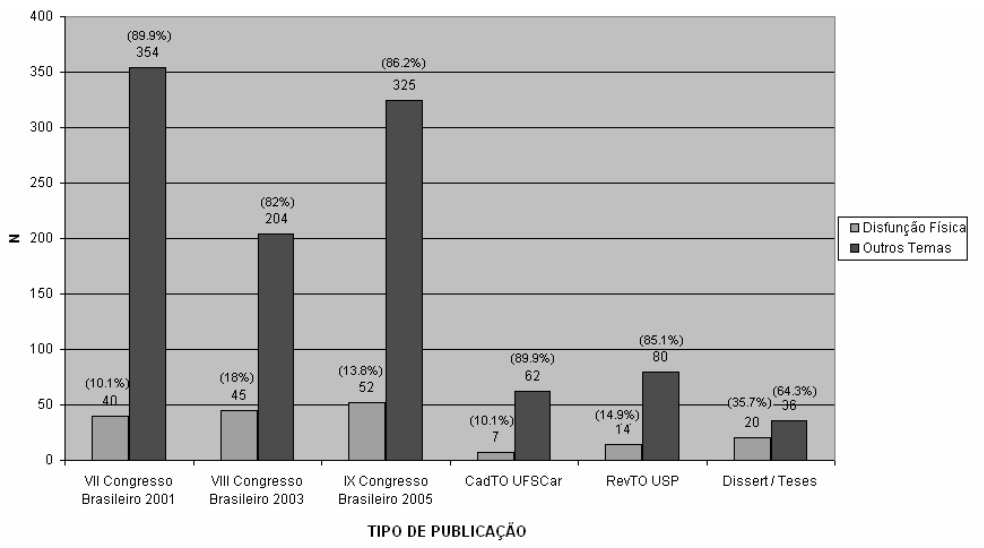

A mesma diferença se faz presente também na representação dos artigos na Revista de Terapia Ocupacional da USP $(14,9 \%)$ e Cadernos de Terapia Ocupacional da UFSCar
$(10,1 \%)$ podendo sugerir menor disponibilidade de autores sobre o tema em apresentarem artigos a revistas da área.

É no campo das teses e dissertações, que existe maior 
participação, onde cerca de $35 \%$ delas trataram sobre o tema deste estudo, podendo representar a preocupação dos profissionais com tema de amplo interesse para a constituição da terapia ocupacional historicamente.
Após a leitura dos textos foram levantadas algumas categorias, indicadas no Quadro 1, que demonstram as preocupações levantadas pelos profissionais ao delinear a apresentação de sua produção bibliográfica.

Quadro 1 - Temáticas apresentadas pelos autores nos textos estudados, segundo tipo de produção bibliográfica.

\begin{tabular}{lcccc}
\hline Temáticas & Resumos & Artigos & Dissertações/Teses & Livros/Capítulos \\
\hline Ampliação de redes sociais & 5 & 7 & 1 & 1 \\
Análise do contexto & 21 & 12 & 3 & 3 \\
Avaliação de programas & 12 & 5 & 3 & 1 \\
Participação da comunidade & 14 & 9 & 3 & 1 \\
Diminuição de barreiras (arquitetônicas/sociais) & 3 & - & 2 & 2 \\
Discussão de modelos e/ou métodos (saúde em geral e TO) & 5 & 6 & 8 & - \\
Intervenções sobre o corpo (capacidade funcional) & 44 & 13 & 7 & 4 \\
Intervenções sobre o corpo (independência/autonomia) & 16 & 12 & 6 & 3 \\
Intervenções sobre o corpo (inserção social) & 1 & 3 & 2 & - \\
Novas tecnologias & 5 & - & 3 & - \\
Saúde mental da pessoa com disfunção física & 21 & 2 & 5 & 1 \\
Enfoque da família & 5 & 2 & 4 & 1 \\
Promoção da autonomia & 19 & 2 & 1 & 6 \\
Revisão bibliográfica & 4 & 5 & 3 & - \\
Ajudas técnicas e equipamentos & - & 7 & 3 & - \\
Inclusão social pelo trabalho & - & 6 & - & \\
\hline
\end{tabular}

Obs: para cada texto estudado foram levantadas diversas categorias procurando descrever a natureza das preocupações dos autores

Entre os resumos apresentados em congressos chamam a atenção as temáticas que buscam lidar com a capacidade funcional, com a saúde mental, com análise do contexto, com a promoção da autonomia e independência, apesar de que estão pouco delineadas as maneiras ou formas de implementar a análise de contexto ou a saúde mental pela necessidade de síntese que esses tipos de produção implicam. Observou-se tendência semelhante nos artigos, dissertações e livros e capítulos para incorporação ainda inicial dessas temáticas.

A questão das ajudas técnicas e equipamentos estão mais presente nos artigos, livros e capítulos, publicações que facilitam a divulgação dessa temática, por seu formato e vocação. Os artigos para divulgar as alternativas em que os profissionais têm buscado pesquisar e os livros e capítulos, por serem obras que comportam textos mais extensos e de formação geral, muitas vezes manuais para a estruturação da prática profissional. Vale lembrar que os livros de terapia ocupacional de autores nacionais são recentes e os que fizeram parte deste estudo representam importante tendência de produção na área nos últimos cinco anos.

Os manuais de terapeutas ocupacionais brasileiros seguem as tendências internacionais de pontuar principalmente as ações sobre a função corporal ao se lidar com pessoas com disfunção física, indicando orientações e procedimentos baseados em patologias mais comuns, mesmo que se indique a necessidade de avaliação do contexto pessoal social, cultural e ambiental como já indicou Trombly (2005)

Entre as dissertações e teses têm importância tanto a discussão e reflexão sobre a origem de modelos e/ou métodos como as intervenções sobre o corpo facilitadoras de independência e autonomia, campos de atividade tradicional de terapia ocupacional em disfunção física. Sendo assim, se observa uma tendência a reflexão que também apareceu nos resumos e artigos publicados no período.

Por outro lado, ficou evidente a preocupação com a ampliação das redes sociais, com a análise de contexto, com a avaliação de programas e participação da comunidade e saúde mental das pessoas com disfunção física dos autores que apresentaram trabalhos em congressos, revelando o surgimento de uma tendência entre os profissionais da área

envolvidos com a prática assistencial e com pesquisas.

Essas questões não estão tão presentes nos livros e capítulos, ainda em pequeno número na área, e, por vezes, cumprindo o papel de manuais ou livros didáticos dedicando-se a instrumentalizar os leitores para lidar com as questões relativas às intervenções especializadas sobre o corpo e sobre o planejamento e confecção de ajudas técnicas e equipamentos.

Em todos os tipos de publicação se apresentaram revisões bibliográficas demonstrando a preocupação em conhecer e analisar tendências para o campo de 
conhecimento, podendo significar maturidade acadêmica dos profissionais.

\section{CONSIDERAÇÕES FINAIS}

Uma das dificuldades encontradas para o levantamento de dados para este estudo foi a exigüidade de informações disponíveis nas bibliotecas das escolas e universidades sendo necessário contar tanto com o levantamento de dados das bases bibliográficas citadas como dos acervos que vão se constituindo pessoalmente, já que a circulação de livros de terapia ocupacional em nível nacional ainda é restrita.

Os terapeutas ocupacionais brasileiros têm apresentado uma produção bibliográfica crescente nos últimos anos, mas as publicações sobre a temática da terapia ocupacional em disfunção física, pela importância histórica que tem na profissão, ainda não representam percentual significativo da produção bibliográfica nacional. Isto poderia demonstrar menor preocupação desses profissionais em participar em eventos da área, em divulgar seus trabalhos nos veículos específicos, ou ainda menor número de profissionais envolvidos com temática tão tradicional da terapia ocupacional.

Nos congressos da área se observaram as preocupações tradicionais de delineamento de alternativas e intervenções sobre o corpo e a função corporal, a promoção da autonomia, ao mesmo tempo em que se ampliaram para a indicação de preocupações com a análise de redes sociais, de contexto, da saúde mental e de avaliação de programas. Essa tendência poderia ser monitorada nos próximos congressos da área, bem como seria produtivo considerar a possibilidade de acessar a produção bibliográfica de profissionais em congressos de áreas afins, pelo menos daqueles vinculados ao ensino e para os quais seria possível encontrar essas informações em bancos de dados disponíveis.

No estudo chamou à atenção a presença da temática nas dissertações e teses, preocupação daqueles que se interessam no desenvolvimento de pesquisa o que pode significar ampliação de estudos nessa área nos próximos períodos, já que mestres e doutores, muito provavelmente, serão os futuros pesquisadores em terapia ocupacional, constituindo corpo teórico e reflexivo para a formação profissional e acadêmica.

Por outro lado, ainda há necessidade de transformar a presença numericamente significativa de trabalhos apresentados em congressos, cerca de $64 \%$ da produção na área em produções bibliográficas de maior repercussão para o campo profissional tendência que deve ser estimulada entre profissionais e estudantes que participem dos próximos eventos da categoria profissional. $\mathrm{O}$ volume de resumos poderia ser acompanhado do aumento de artigos e capítulos de livros, que aprofundariam as reflexões trazidas auxiliando na reflexão sobre fundamentação das atividades profissionais.

Há necessidade de ampliar os esforços para maior divulgação e circulação da produção bibliográfica da área.

FERREIRA, T. G.; OLIVER, F. C. Occupational therapy in physical dysfunction: discussing the brazilian bibliographical production from 1999 to 2005. Rev. Ter. Ocup. Univ. São Paulo, v. 17, n. 3, p. 108-114, set./dez., 2006

\begin{abstract}
This is a study of the Brazilian bibliography regarding occupational therapy in physical dysfunctions based on thesis, dissertations, articles and abstracts presented in conferences between 1999 and 2005. We consulted: LILACS - Latin American and Caribean Literature on Health Science, SciELO Scientific Eletronic Library Online and the thesis achieves at CAPES. In regards to the abstracts, we searched the annals of the VII and IX Brazilian Occupational Therapy Conference and the VIII Brazilian and V Latin American Occupational Therapy Conference. We also identified books and manuals in this area of interest, edited between 2001 and 2005. For the articles we also searched the Cadernos de Terapia Ocupacional of the Federal University of São Carlos. There were found 213 texts about this theme. In the considered universe the abstracts presented in conferences represented $64 \%$ of the production while the articles, thesis and books represented, respectively, 20\%, 9\% and 7\%. Regarding the published abstracts we observed a growth tendency in the given period, which did not occur with the articles. These had a rise between 1999 and 2002 and later decreased with the rise of publishing of books. The majority of the thesis and dissertations where published in 1999, 2003 and 2004. Debate on the intervention for functional capacity/ motor skills/independence/autonomy appears as a core theme, as well as concerns for the analysis of context, mental health of the person with disability, methods of intervention, among others.
\end{abstract}

KEY WORDS: Occupational therapy. Bibliography (Publication type). Areas of intervention in occupational therapy. Disabled persons/rehabilitation. 
FERREIRA, T. G.; OLIVER, F. C. Terapia ocupacional em disfunção. Rev. Ter. Ocup. Univ. São Paulo, v. 17, n. 3, p. 108-114, set./dez., 2006.

\section{REFERÊNCIAS}

BARROS, D. D.; OLIVER, F. C. Contribuindo para a discussão do Qualis de Terapia Ocupacional no Brasil. Rev. Ter. Ocup. Univ. São Paulo, v. 14, p. 52-62, 2003.

GIL, A. C. Como elaborar projetos de pesquisa. 3a ed. São Paulo: Atlas, 1996.

PÁDUA, E. M. M. Metodologia de pesquisa: abordagem teóricoprática. 7a ed. Campinas: Papirus, 2002.
TROMBLY, C. A.; RADOMSKI, M. V. Terapia ocupacional para disfunções físicas. 5a. ed. Santos: Ed Santos, 2005.

WORLD FEDERATION OF OCCUPATIONAL THERAPISTS - WFOT. What is occupational therapy? Disponível em: http://www.wfot.org.au. Acesso em: 22 jun. 2005.

Recebido para publicação: Set./2006

Aceito para publicação: Out./2006 